PROCEEDINGS OF THE

AMERICAN MATHEMATICAL SOCIETY

Volume 129, Number 10, Pages 2955-2957

S 0002-9939(01)05909-3

Article electronically published on March 14, 2001

\title{
A REMARK ON A PAPER OF E. B. DAVIES
}

\author{
MACIEJ ZWORSKI
}

(Communicated by Christopher D. Sogge)

Abstract. We explain the existence of open sets of complex quasi-modes in terms of Hörmander's commutator condition.

In a recent paper [1, Davies proves the following interesting result: if $P(h)=$ $\left(h D_{x}\right)^{2}+V(x), D_{x}=(1 / i) d / d x, V \in \mathcal{C}^{\infty}(\mathbb{R})$, then

$$
\begin{gathered}
\operatorname{Im} V^{\prime}\left(x_{0}\right) \neq 0, \quad E=\xi_{0}^{2}+V\left(x_{0}\right), \xi_{0} \in \mathbb{R} \backslash\{0\} \Longrightarrow \\
\exists u(h) \in L^{2}(\mathbb{R}),\|u(h)\|_{L^{2}(\mathbb{R})}=1,(P(h)-E) u(h)=\mathcal{O}\left(h^{\infty}\right),
\end{gathered}
$$

where $\mathcal{O}\left(h^{\infty}\right)$ is a bound in any norm, for instance $\mathcal{C}^{k}$ for any $k$.

The purpose of this note is to point out that a more general statement than (1) follows immediately from the now standard results in microlocal analysis described in Chapter 26 of [5].

Let $X$ be a manifold and $S^{m, k}\left(T^{*} X\right)$ be the space of semi-classical symbols on $T^{*} X: a \in \mathcal{C}^{\infty}\left(T^{*} X \times(0,1]\right)$,

$$
\partial_{x}^{\alpha} \partial_{\xi}^{\beta} a(x, \xi ; h) \leq C_{\alpha, \beta} h^{-m}(1+|\xi|)^{k-|\beta|}, \quad(x, \xi) \in T^{*} X, h \in(0,1] .
$$

The local quantization formula

$$
a\left(x, h D_{x} ; h\right) u=\frac{1}{(2 \pi h)^{n}} \iint a(x, \xi ; h) e^{i\langle x-y, \xi\rangle} u(y) d y d \xi
$$

defines a class of semi-classical pseudo-differential operators on $X, \Psi_{h}^{m, k}(X)$, with a symbol map, $\sigma$, and a short exact sequence,

$$
0 \longrightarrow \Psi_{h}^{m-1, k-1}(X) \longrightarrow \Psi_{h}^{m, k}(X) \stackrel{\sigma}{\longrightarrow} S^{m, k}\left(T^{*} X\right) / S^{m-1, k-1}\left(T^{*} X\right) \longrightarrow 0 .
$$

If we strengthen the condition (2) to

(3) $\left(h D_{h}\right)^{l} \partial_{x}^{\alpha} \partial_{\xi}^{\beta} a(x, \xi ; h) \leq C_{l, \alpha, \beta} h^{-m}(1+|\xi|)^{k-|\beta|}, \quad(x, \xi) \in T^{*} X, h \in(0,1]$,

we can then use, without any modifications, the $\mathcal{C}^{\infty}$ theory of pseudo-differential operators when working in compact subsets of $T^{*} X$. We can simply introduce a new variable $t \in \mathbb{R}$ and consider $h=1 / \tau$, where $\tau$ is the dual variable to $t$. Working microlocally in the region $|\xi / \tau| \leq C$ corresponds to working semi-classically in compact subsets of $T^{*} X$. In particular we have a correspondence between the

Received by the editors January 25, 2000 and, in revised form, February 14, 2000.

2000 Mathematics Subject Classification. Primary 35Sxx, 81Q20.

(C)2001 American Mathematical Society 
frequency set and the wave front set:

$$
\begin{aligned}
& (x, \xi) \notin W F_{h}(u(h)) \Longleftrightarrow \\
& \left\{\begin{array}{l}
\exists Q \in \Psi^{0}(\mathbb{R} \times X) \text { elliptic at }(t, x, \tau, \tau \xi) \text { for all }(t, \tau) \in T^{*} \mathbb{R} \backslash 0 \\
\text { such that } Q \mathcal{F}_{\tau \mapsto t}^{-1}(u(1 / \bullet)) \in \mathcal{C}^{\infty}(\mathbb{R} \times X),
\end{array}\right.
\end{aligned}
$$

where $\mathcal{F}_{\tau \mapsto t}^{-1}$ is the inverse Fourier transform (see [3] and [6. Proposition 9] for the definition and the relation to other wave front sets). The characterization given here follows from the one in $[6]$.

This allows us to adapt directly the results of Hörmander and of Duistermaat and Sjöstrand (see [2] and [5, Sect.26.3]) to obtain the following statement: if $P\left(x, h D_{x} ; h\right)$ is a semi-classical pseudo-differential operator, in the class satisfying (3), and with the principal symbol $p$, then

$$
\begin{gathered}
p(m)=0, \quad\{\operatorname{Re} p, \operatorname{Im} p\}(m)<0, \quad m \in T^{*} X \Longrightarrow \\
\exists u(h) \in \mathcal{C}^{\infty}(X) \text { such that } W F_{h}(u(h))=\{m\} \text { and } W F_{h}(P u)=\emptyset .
\end{gathered}
$$

If we take $P\left(x, h D_{x} ; h\right)=\left(h D_{x}\right)^{2}+V(x)-E$ we immediately recover Davies's result: if $\chi \in \mathcal{C}_{\mathrm{c}}^{\infty}(X)$ is equal to 1 near $x_{0}, m=\left(x_{0}, \xi_{0}\right)$, then, because of the $W F_{h}$ statements, $P(\chi u)=\mathcal{O}\left(h^{\infty}\right)$ and $\chi u \in \mathcal{C}_{\mathrm{c}}^{\infty}(X)$. The condition on the Poisson bracket generalizes the one dimensional condition $\operatorname{Im} V^{\prime}\left(x_{0}\right) \neq 0$. For Schrödinger operators in higher dimensions, we need $E=\xi_{0}^{2}+V\left(x_{0}\right), \operatorname{Im} \xi_{0} \cdot \nabla V\left(x_{0}\right) \neq 0$.

We conclude with a few remarks. The microlocal statement (4) is a generalization of the celebrated commutator condition of Hörmander 4. The crucial part of his argument was in fact a geometric optics "quasi-mode" construction which, in a very special case, is repeated in [1. As was pointed out to the author by Victor Ivrii, the microlocal commutator condition (4) has the following well known global analogue: let $Q$ be an unbounded operator on a Hilbert space $\mathcal{H}$, with the domain $\mathcal{D}$. Let us also assume that $Q^{*}$ has the same domain as $Q$ and that $Q-z, Q^{*}-\bar{z}: \mathcal{D} \rightarrow \mathcal{H}$, are Fredholm operators for all $z$. Then

$$
\pm\left[Q, Q^{*}\right] \geq C>0 \Longrightarrow \operatorname{spec} Q=\mathbb{C} .
$$

In the case of the $+\operatorname{sign}, Q-z$ fails to be injective for all $z$, and in the case of the - sign, surjective. In fact, we conclude that for all $z \in \mathbb{C}$ and $u \in \mathcal{D}$,

$$
\mp\|(Q-z) u\|^{2} \pm\left\|\left(Q^{*}-\bar{z}\right) u\right\|^{2} \geq C\|u\|^{2} .
$$

If $Q-z_{0}$ were invertible at some $z_{0}$, then index $(Q-z)=\operatorname{index}\left(Q-z_{0}\right)=0$. In the - case $Q-z$ would then be invertible everywhere, and hence the spectrum is either empty or equal to $\mathbb{C}$. In the + case we draw the same conclusion for the adjoint.

We finally remark that, as pointed out by Davies, the existence of complex quasi-modes implies lower bounds on the resolvent, and that has computation consequences.

\section{ACKNOWLEDGMENTS}

I should like to thank Fréderic Klopp and Victor Ivrii for helpful discussions and Steve Zelditch for encouraging me to publish this note. 


\section{REFERENCES}

[1] E.B. Davies, Semi-classical states for non-selfadjoint Schrödinger operators, Comm. Math. Phys. 200(1999), 35-41. MR 99m:34197

[2] J.J. Duistermaat and J. Sjöstrand, A global construction for pseudo-differential operators with non-involutive characteristics, Inv. Math. 20(1973), 209-225. MR 49:9681

[3] V.W. Guillemin and S. Sternberg, Geometrical Asymptotics, Mathematical Surveys, 14, Amer. Math. Soc., Providence, R.I., 1977. MR 58:24404]

[4] L. Hörmander, Differential equations without solutions, Math. Ann. 140(1960), 169-173. MR 26:5279

[5] L. Hörmander, The Analysis of Linear Partial Differential Operators IV, Grundlehren der mathematischen Wissenschaften 275, Springer-Verlag, 1985. MR 87d:35002b

[6] R.B. Melrose and M. Zworski, Scattering metrics and geodesic flow at infinity, Invent. Math. 124(1996), 389-436. MR 96k:58230

Department of Mathematics, Evans Hall, University of California, Berkeley, CaliFORNIA 94720

E-mail address: zworski@math.berkeley.edu 